\title{
Three-Dimensional Visualization of Herpesvirus Envelopment at High Resolution Using STEM Tomography and Serial Sectioning on High Pressure Frozen/Freeze- Substituted Cells
}

\author{
Martin Schauflinger ${ }^{1}$, Clarissa Villinger ${ }^{2,3}$, Jens von Einem ${ }^{2}$ and Paul Walther ${ }^{3}$. \\ 1. Electron Microscopy Core Facility, University of Missouri, Columbia, Missouri, USA. \\ 2. Institute of Virology, University Medical Center Ulm, Ulm, Germany. \\ 3. Central Facility for Electron Microscopy, Ulm University, Ulm, Germany.
}

Human cytomegalovirus (HCMV) is a clinically highly relevant Herpesvirus. Lacking a suitable animal model, HCMV's strict species specificity is a major obstacle for the characterization of biological and pathogenic viral phenotypes. Electron microscopy is a suitable tool to characterize viral phenotypes, unraveling viral morphogenesis. We used advanced EM technologies to visualize the HCMV final envelopment processes in the viral assembly complex. Three-dimensional visualization using STEM tomography and serial sectioning was used to study HCMV final envelopment at high resolution.

Infected cells were rapidly cryo-immobilized by high pressure freezing in order to overcome the disadvantages of traditional chemical fixation. High pressure freezing in combination with freeze substitution grants superior preservation of subcellular structures and is favorable for the examination of dynamic processes like virus envelopment. STEM tomography was applied as described [1] to visualize virus-vesicle interactions with high resolution (Fig. 1). A limiting disadvantage of STEM tomography is that only a relatively small volume (the $\mathrm{Z}$ height is limited to $\sim 1$ micron) of the infected cell can be imaged.

The more traditional method of serial sectioning was used for three dimensional visualization of the whole HCMV assembly complex [2] (Fig. 2). The viral assembly complex is the specific area in the cytoplasm of HCMV infected cells in which final envelopment of viral particles occurs. The assembly complex exhibits distinct local membrane compositions: early endosomes in its central region, and vesicles characterized by Golgi markers in its periphery. This led us to speculate that budding events might occur preferentially in either the central or the peripheral area of the assembly complex.

Using the 3D high resolution afforded by both STEM tomography and serial sectioning on high pressure frozen HCMV-infected cells, it was possible to determine the distribution of enveloped and non-enveloped virus particles throughout the area of the assembly complex. Contrary to our hypothesis, quantitative analysis revealed that the events of final envelopment are equally distributed within the assembly complex irrespective of the local membrane composition $[2,3]$.

\section{References:}

[1] Villinger et al. in "Electron Microscopy", ed. J Kuo (Humana Press, Totowa), pp. 617-638.

[2] Schauflinger M, Villinger C, Mertens T, Walther P, and von Einem J. Cell Microbiol 15 (2013), 305-314.

[3] This work was supported by the DFG priority research program SPP1175. 


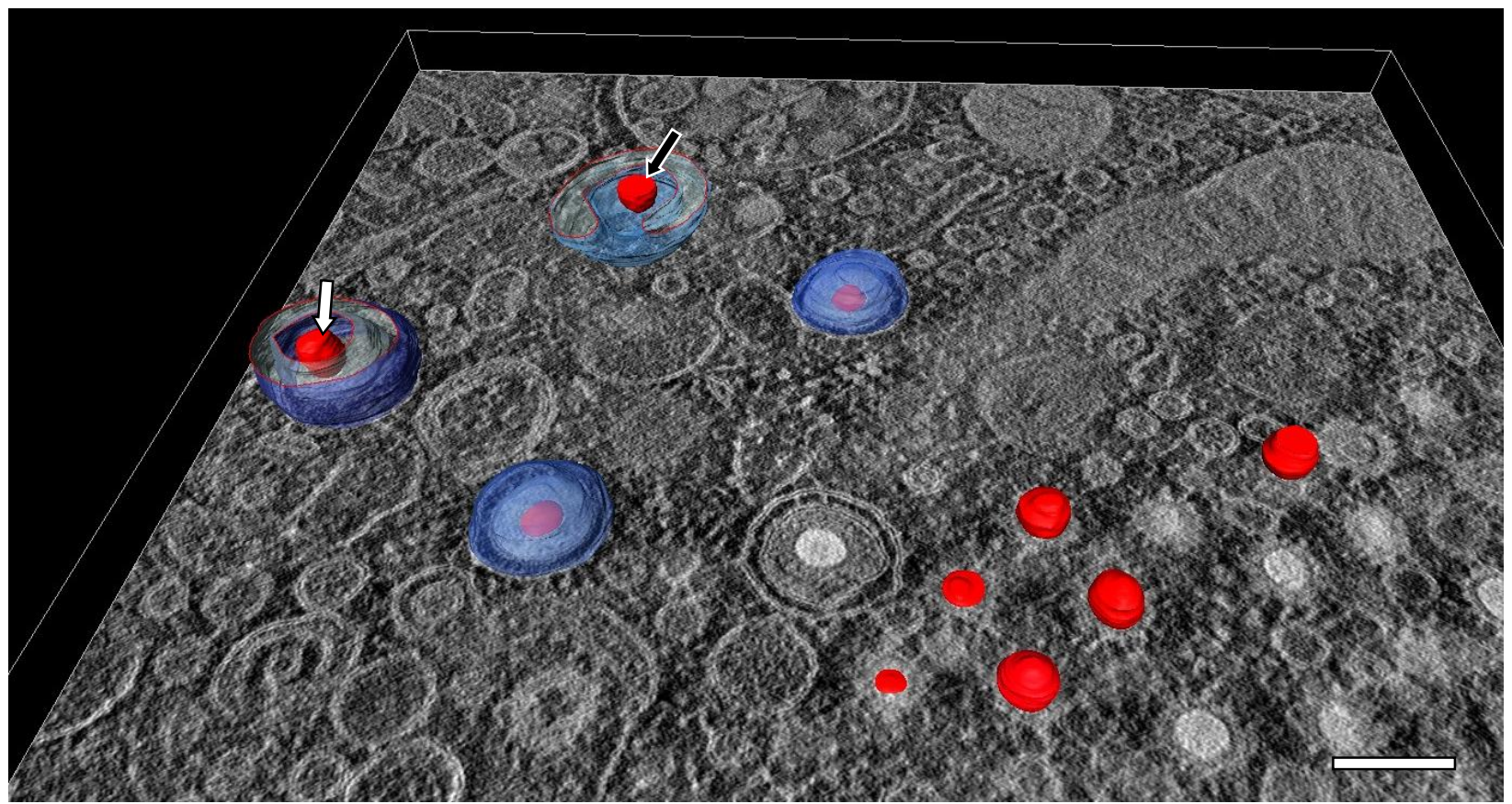

Figure 1. Three-dimensional reconstruction of membranes and viral capsids from STEM tomography of HCMV-infected fibroblasts at 5 days post infection. Areas with high density, such as membranes and capsids, occur bright in the dark-field STEM tomogram. HCMV capsids before (lower right), during (black arrow), and after (white arrow) final envelopment were reconstructed and superimposed on a single slice of the tomogram. The leaflets of viral membranes are depicted in blue and grey respectively; viral capsids are indicated in red. Red lines indicate the cut-away plane in which the segmented structures have been revealed to better visualize the membranes around the capsids. Bar $200 \mathrm{~nm}$.

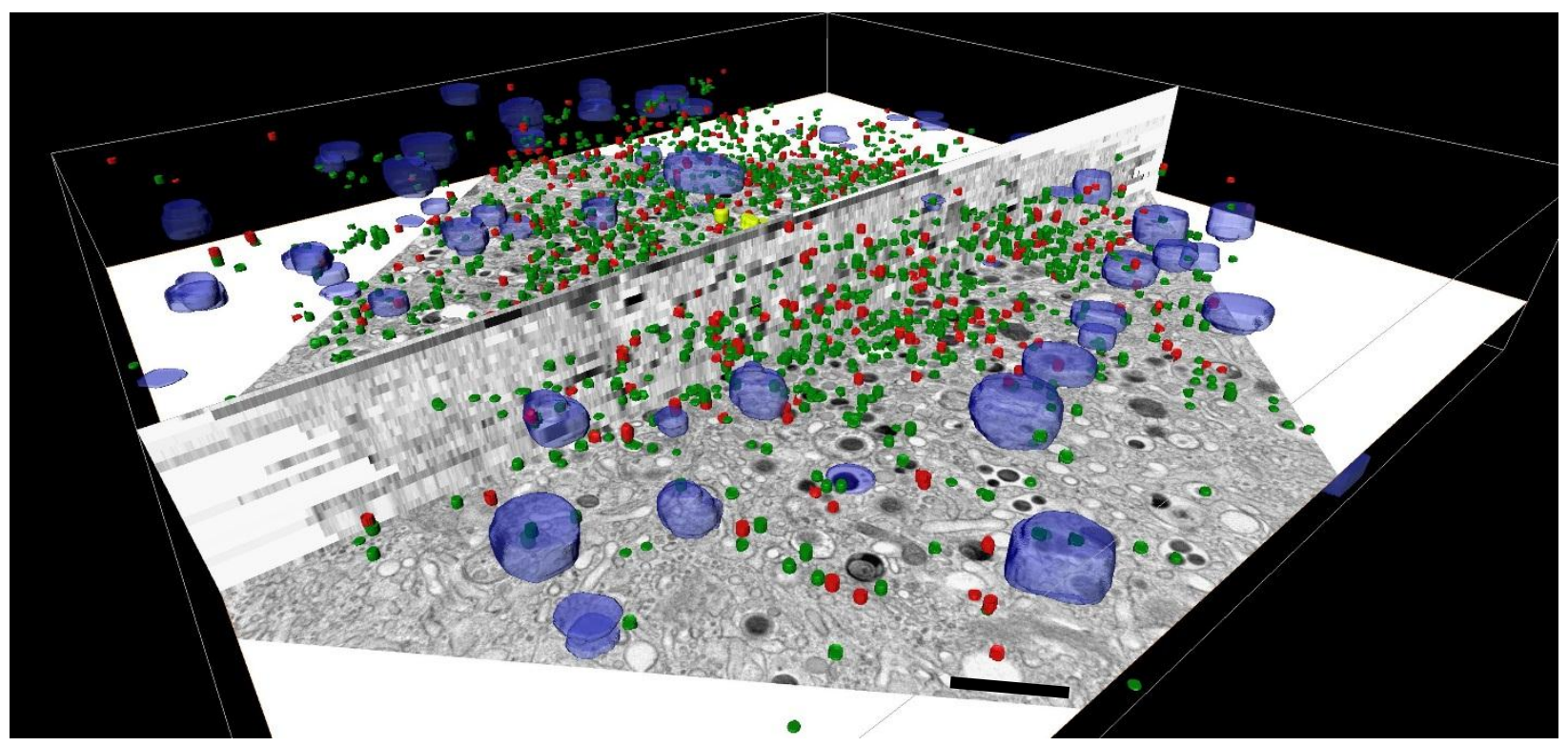

Figure 2. Micrographs from the HCMV assembly complex were taken with a nominal magnification of $12,000 \mathrm{x}$ from a total of 28 sections and aligned into an image stack. Shown here is a single micrograph in $\mathrm{X}-\mathrm{Y}$ direction, while the image stack is indicated by a slice through the Z-axis. Cellular and viral structures were segmented on all sections and superimposed. Enveloped virions (green), non-enveloped particles (red), multivesicular bodies (transparent blue). Bar 1 um. 